\title{
Comics und graphic novels im multikulturellen Deutschunterricht
}

\section{Comics and graphic novels in multicultural German-language-lessons}

\begin{abstract}
This essay examines how comics and graphic novels can be used in multicultural German-language-lessons. It demonstrates the correlation between visual literacy and reading comprehension and shows different methods - analytical, productive and creative. Furthermore it focuses on the language of comics and on their semiotic diversity.

KEYWORDS: comics, graphic novels, visual literacy, reading comprehension, methods, semiotic diversity, icons, symbols, indices.
\end{abstract}

\section{POTENZIALE DER GRAPHIC NOVELS UND COMICS IM DEUTSCHUNTERRICHT}

Im Deutschunterricht gehören Comics und graphic novels noch nicht unbedingt zu den kanonischen Beständen, obwohl sie im Laufe der Jahrzehnte das Stigma des scheinbar Minderwertigen ablegten und sich als sequential art (Eisner 1998) oder invisible art (McCloud 2001) etablierten. Als Teil kulturellen Praxis ermöglichen sie Eskapismus und Floating-Erlebnisse, Alteritätserfahrungen, fördern Empathie und Persönlichkeitsentwicklung, verhelfen zum deklarativen Wissen, zum ästhetischen Genuss und zu den Gratifikationserlebnissen und weisen sowohl individuelle als auch soziale Bedeutsamkeit auf. Sie sind mit historischen Situationen verbunden und können als Symbolträger und kulturelle Erinnerungsmarker betrachtet wer- 
den. Sie reflektieren gesellschaftliche Probleme, bringen (fremde) Kulturen nahe, decken die gesamte Themenpalette der fiktionalen Texte ab und laden zum Perspektivenwechsel ein. Wolfgang Hallet spricht in diesem Sinne von graphic novels als einem kulturellen Versuch, die oft schwer durchzuschaubaren gesellschaftlichen Prozesse, die entscheidenden historischen Erfahrungen, die kulturellen Entwicklungen und die technologischen Revolutionen visuell $\mathrm{zu}$ repräsentieren, $\mathrm{zu}$ strukturieren und erfahrbar $\mathrm{zu}$ machen (Hallet 2012: 3).

Ihr Potenzial für den gegenwärtigen kompetenzorientierten Deutschunterricht ist groß. So können Comics und graphic novels zur Entwicklung und Förderung von Lese- und Schreibkompetenz, von lexikalischen, sprachreflexiven und interkulturellen Kompetenzen sowie zur Ausbildung eines differenzierten Medienverständnisses herangezogen werden. Sie üben eine motivierende Wirkung aus und können den jungen Lesern einen Einstieg in die Werke von Klassikern geben. Um hier lediglich einige Beispiele zu geben Horus 2005 und Kreitz 2009 und 2012. Es sollte allerdings nicht dabei belassen werden.

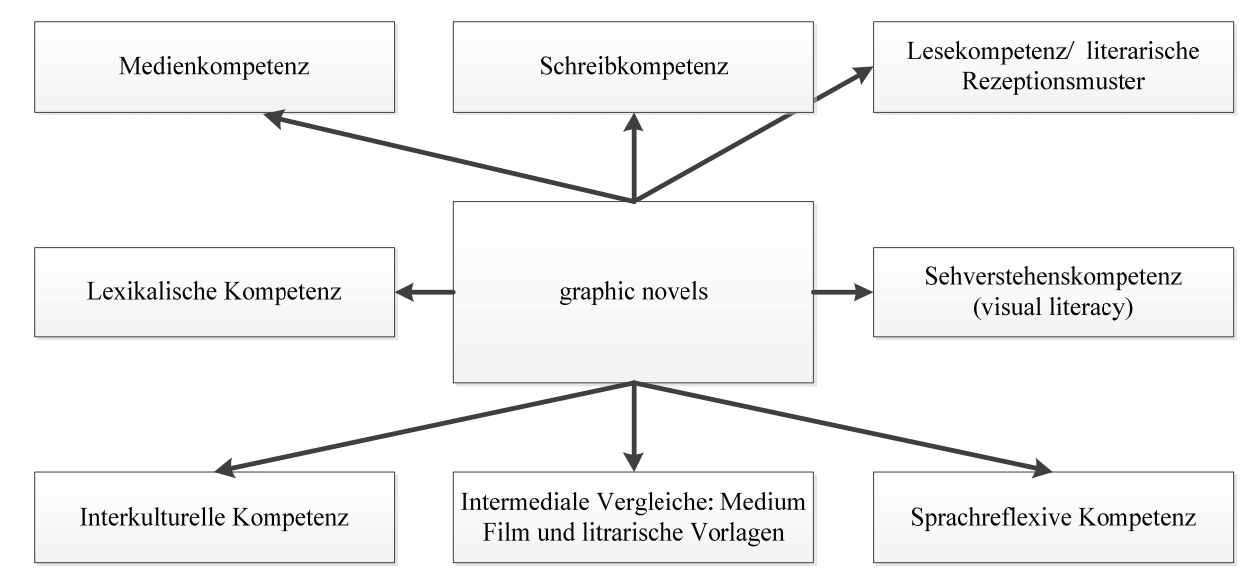

Potenziale der graphic novels im Deutschunterricht (Eigene Darstellung)

Die für das Medium spezifischen reduzierten verbalsprachlichen Textanteile sind leichter zu bewältigen, vor allem dann, wenn Bild und Erzähloder Dialogtext sich komplementär zu einander verhalten. Dadurch werden sowohl den monolingual deutschsprachigen Schülern als auch den Lernenden mit einer anderen sprachlichen und kulturellen Herkunft zusätzliche Impulse und Anhaltspunkte für die Sinnkonstruktion der textuell-grafischen Vorlagen und konsequenterweise eine aktive Beteiligung am Unterrichtsge- 
schehen ermöglicht. Der grafische Kontext - die denotativen und die konnotativen Bedeutungen der visuellen Darstellungen - kann selbst zum Gegenstand des Unterrichts gemacht werden. Ein weiterer Aspekt, der bei der Einbeziehung von Comics und graphic novels eine Berücksichtigung finden sollte, der allerdings das Beherrschen eines Analyse-Instrumentariums voraussetzt, um diverse Phänomene der Comicsprache auf der Metaebene benennen zu können, ist ihre Machart. Die Beherrschung dieses Instrumentariums macht die Wirkung durchschaubar und diskutierbar. Auch der Rezeptionsvorgang im Sinne eines kompetenten Umgangs mit den visuellen Narrationen sollte zum Gegenstand des Unterrichts gemacht werden. Dies wäre ein gelungener Beitrag zur Leseförderung.

\section{REZEPTIONSVORGÄNGE BEI DER LEKTÜRE VON GRAPHIC NOVEL}

Die Besonderheit der Lektüre von graphic novels und Comics impliziert die Lesemuster. So verlangt die westliche Konvention den Beginn der sequenziellen Rezeption von panels in der oberen linken Ecke. Mangas (Japan), Manhwas (Südkorea) und Manhuas (China) verlangen dem Lesenden andere Vorgehensweisen ab: von hinten nach vorn und demzufolge von rechts oben nach links unten. Der Lese- und Verstehensvorgang beginnt jedoch unabhängig von der Herkunft der literarischen Vorlage mit dem Verschaffen des ersten Gesamteindrucks, mit dem Erkennen des Textes als ein Comic und mit der "Erwartung einer Geschichte“ oder in der Terminologie von Teun van Dijk und Walter Kintsch mit der "Aktivierung des narrativen Schemas" (Dijk, Kintsch 1983: 56ff.). Dabei können auch Merkmale wie Formen oder Farben oder Inhalt der einzelnen panels die Aufmerksamkeit des ersten Blickes auf sich ziehen. Auch die Identifikation des Zeichentyps, beispielsweise Super-Held-Comic spielt eine Rolle.

Comics und graphic novels als moderne Varianten des Prinzips Bildergeschichte sind visuelle Narrationen, die zwar keine normativen Regeln über die Art und Weise, wie sie Geschichten erzählen, aufstellen lassen, doch können zwei Elemente als konstituierend bestimmt werden: die Interdependenz und Gleichwertigkeit der Bilder/ Zeichnungen und Texte und das Vorhandensein von mindestens zwei panels, die durch eine Lücke (gutter/ Hiautus) voneinander getrennt sind und zu einer Bedeutungseinheit (globale Kohärenz) kombiniert werden. Der Leser wird somit versuchen in der Übereinkunft mit der Geschichts-Struktur eine globale Kohärenz zwischen einzelnen Elementen oder Zeichen zu kreieren. Dies setzt zunächst das Bilden von Inferenzen zwischen den einzelnen panels voraus. Daraus 
ergeben sich weitere Besonderheiten für die Rezeption: die Lesenden müssen kontinuierlich zwischen der Rezeption der Bilder und des Textes wechseln, d.h. der Leser kann nicht innerhalb eines Modus bleiben, um beispielsweise zunächst den ganzen Text auf einer Seite zu lesen und danach die Bilder zu rezipieren, um die Comic verstehen zu können. Die Handlung erschließt sich dem Leser nur aus der Betrachtung beider Elemente. Das Bilden von Inferenzen geht mit der Verknüpfung der einzelnen visuellen und textuellen Elemente mit den bereits vorhandenen Wissensbeständen einher. Es herrscht mitunter ein Konsens, dass das Lesen eines Textes einen interaktiven Vorgang darstellt, bei dem von Anbeginn der Lektüre und so lange bis keine textuellen Impulse mehr vorliegen, textgeleitete Verfahren (bottom up) und wissensgeleitete Komponenten (top down) miteinander interagieren. Der Leser stellt kontinuierlich Hypothesen auf - vorläufig erfolgte Bedeutungsgebung (Karg, Kuzminykh 2014) - die er im Laufe des Lesevorgangs mit neuen Informationen ergänzt, revidiert oder aber auch verwirft, und auf diese Art seine Sinnzumessung entfaltet. Kognitionspsychologisch formuliert erstellt der Lesende ein Situationsmodell des Gelesenen, d.h., er entwickelt eine Vorstellung von dem, wovon der Text handelt (Kintsch 1998). Das Wissen, das in Sozialisation- und Enkulturationsprozessen vermittelt bzw. angeeignet wird, stellt dabei eine unabdingbare Voraussetzung für den Verstehensvorgang dar und kann als eine Disposition betrachtet werden. In der hermeneutischen Tradition wird in diesem Zusammenhang von einem Vorurteil gesprochen (Gadamer 1990). Das Ergebnis des Gelesenen muss zwar verhandelbar bleiben, darf dennoch nicht willkürlich sein. D.h., das Ergebnis des Leseverstehensvorgangs ist in einen Kontext einzubauen, der zusätzliche Erkenntnisse vermittelt und Akzentuierungen ermöglicht. Das Leseinteresse und die vor der Lektüre gestellten Ziele sind für den Leseverstehensvorgang konstituierend.

Die Rezeption der Bilder ist mit dem Beschriebenen vergleichbar. Auch hier geht der Rezipient zunächst von den visuellen Signalen aus, interpretiert diese und misst ihnen Bedeutung zu. Der Prozess der Sinnzuweisung wird als visual literacy (Sehverstehenskompetenz) bezeichnet und impliziert die Fähigkeit, die im Bild präsentierte Welt zu lesen und zu deuten. Es wird differenziert zwischen dem kulturellen Sehen, das Aktionen, Interaktionen, soziale Konstellationen, Funktion von Gegenständen umfasst und dem ästhetischen Sehen (der bildästhetischen Kompetenz), das die Formensprache von Bildern erfasst (Hallet 2010; Schüwer 2008). Auch dabei ist das Wissen konstituierend. Für die Beschreibung des Sehverstehensprozesses bietet sich neben dem kognitionspsychologischen discourse comprehension model (Kintsch 1998) der Zugriff auf das triadische Zeichensystem von Charles 
Sanders Pierce an. Er geht davon aus, dass das Zeichen sich durch die irreduzible Triade des Representamen, einem Objekt und einen Interpretant konstituiert. Entscheidend für den unterrichtlichen Kontext ist jedoch die Trichotomie von Ikon, Index und Symbol als Verbindung des Zeichens mit dem Objekt. Das Ikon verweist mit seinen Eigenschaften auf das dargestellte Objekt und impliziert dadurch einen hohen Grad an Denotation. Dies erklärt, warum Bilder zu einem bestimmten Grad sprachunabhängig und transkulturell verstanden werden können, wenngleich dabei kulturelle Implikationen nicht bzw. unzureichend interpretiert werden. Die Ikonizitätsgrade können variieren beispielsweise durch das Verfremden der Realitätsobjekte oder durch eine ungewöhnliche farbliche Darstellung. Die gezeichneten Figuren in den Comics lassen sich durchaus als Ikonen im Sinne einer materiellen Ähnlichkeit mit Objekten - menschlichen oder tierischen Wesen - erkennen. Das Ikon lässt sich in drei weitere Zeichentypen unterteilen (die hier allerdings nicht weiter berücksichtigt werden können): Bild, Diagramm und Metapher (Pierce 1931-1958: 2277). Auch in Unterricht wäre diese Unterteilung aufgrund ihres abstrakten Charakters weniger sinnvoll.

Der Index wird durch eine kausale Beziehung zum Objekt klassifiziert. Symbole beruhen dagegen auf Konventionen. Alle drei Typen besitzen einen denotativen Aspekt, der zwar unterschiedlich ausgeprägt ist, dennoch nicht $\mathrm{zu}$ bestreiten ist. Denn ein Objekt ist zunächst immer er selbst bevor er zusätzliche konnotative Bedeutung aufnimmt. Die Schwierigkeit erweist sich darin, den spezifischen Mehrwert von Indices und Symbolen zu bestimmen und in den changierenden Deutungsmöglichkeiten ihren Sinn festzulegen. Die visuellen Darstellungen von graphic novels bestehen aus einer Mischung heterogener ikonischer, symbolischer und indexalischer Zeichen, die Ideen und Inhalte jenseits der denotativen Ebene ermöglichen. Diese Zeichen interagieren in einer Weise, die eine klare Trennung zwischen ikonischen, indexalischen und symbolischen Zeichen in Frage stellt. Der Titel kann beispielsweise als symbolisch angesehen weisen, da Kenntnisse des spezifischen Sprachcodes für sein Verständnis notwendig sind und er einen Aufschluss über die Handlung der Geschchte geben sollte. Diese Eigenschaft wird als eine Grundlage für die antizipierende Lesestrategie verwendet (Gold 2007). Er kann aber auch in dieser verweisenden Funktion als ein indexalisches Zeichen interpretiert werden. Symbolische Funktion kommt der Farbgebung, Soundeffektzeichen, von speedlines/Geschwindigkeits- Bewegungslinien und anderen ähnlichen Zeichen zu. Jedes Detail kann bedeutungskonstituierend sein.

Darüber hinaus ist für die Lektüre und für die unterrichtliche Arbeit mit Comics und graphic novels das Konzept der Leerstellen bedeutend (Iser 
1975). Der Leser bezieht die einzelnen Elemente der erzählten Handlung aufeinander, schließt die Leerstellen entsprechend seinen Dispositionen, stellt lokale und globale Kohärenz her und entwirft ein Situationsmodell des Gelesenen bzw. nimmt eine Sinnzuweisung vor. Die Leerstellen verlangen eine aktive Rezeptionsleistung vom Leser ab - es ist ein Wechselspiel, ein Interaktionsprozess zwischen dem Leser und dem Text. Eine ähnliche Funktion kommt den gutters zu. Auch hier ist die Leistung seitens des Rezipienten, einzelne panels als bedeutungshaltige Bildsequenzen aufeinander $\mathrm{zu}$ beziehen und sie in einen logischen Zusammenhang $\mathrm{zu}$ bringen entscheidend. In der Comic-Forschung werden unterschiedliche Arten von panels, gutters und balloons mit einer plotkonstituitiven Funktion bestimmt: plot-, character-, setting-, conflict-, rising-action-, climax- und resolution-panels, moment-to-moment, action-to-action, subject-to-subject, scene-to-scene-gutter, story-, thought, dialogue- und sound-effect-balloons (Monnin 2009). Man kann aber auch Ulf Abraham folgend von zeitlichen, räumlichen, mimischen und gestischen Leerstellen sprechen. Zwischen den einzelnen panels kann Zeit vergangen sein oder die räumliche Umgebung hat sich geändert, ohne dass ein konkreter Hinweis erfolgt ist (Abraham 2012). Der Lesende muss diese Veränderungen wahrnehmen, ihnen einen Sinn zuweisen und schließlich zu einer Verstehensleistung gelangen, die, wie oben bereits erwähnt worden war, durchaus verhandelbar, jedoch nicht willkürlich sein darf.

\section{SPRACHE DER GRAPHIC NOVELS IN DEUTSCHUNTERRICHT}

Die sprachliche Gestaltung von graphic novels und Comics mit typischen Phänomenen der Reduktion und Vereinfachung verdient einer besonderen Zuwendung im Rahmen der Förderung sprachreflexiven Kompetenzen. In den 1970er-Jahren potenzierte sie kritische Einstellungen: Neben den eskapistischen, politisch-suggestiven und chauvinistischen Inhalten verurteile man die Sprache als zu simpel bestehend aus Krüppelsätzen, Ausrufen und Lautnachahmungen und kennzeichnend durch den deiktischen Sprachgebrauch, in dem sich die Bedeutung erst über den situationsspezifischen Kontext erschließt (Welke 1972: 7ff). Man ging komparatistisch vor und exkludierte die Spezifik des Mediums Comic. Es sei an dieser Stelle angemerkt, dass der Vergleich mit anderen Medien, Genres und Modalitäten durchaus legitim ist, nicht aber wenn diese als ein wertender Maßstab herangezogen werden. Anders formuliert, dürfen diverse Medien nicht gegeneinander 
ausgespielt werden, sondern sind in ihrer jeweiligen Spezifik zu würdigen und als gleichwertig zu betrachten.

Nach der Aufwertung des Genres in den 1980er-1990er Jahren zog man positive Rückschlüsse im Hinblick auf das Verstehen der sprachlichen Vorlagen und sah Potenziale für den Sprachunterricht, insbesondere für den fremdsprachlichen (Sanchez-Stockhammer 2012). Folgende 'Forschungsfragen’ sind für die Betrachtung im unterrichtlichen Kontext ergiebig:

\subsection{Konventionen der Comicsprache}

Der Schriftzug erscheint in Comics sowohl in Sprech- und Denkblasen als auch in Form von Anmerkungen am oberen oder seltener unteren Seitenrand (dies in der Regel als Erzählerkommentare). Man kann in diesem Zusammenhand von diegetischen und nicht-diegetischen Elementen sprechen. Das in den Sprechblasen Abgedruckte ist als wörtliche Rede der Figuren aufzufassen, in der Regel in Form eines Dialogs mit anderen. Somit handelt sich hier um die konzeptionelle Mündlichkeit (Koch, Oesterreicher 1985). Es bietet sich an, neben der Thematisierung der Nähe zur konzeptionellen Mündlichkeit intermediale Vergleiche zu Kommunikation in sozialen Netzwerken vorzunehmen, in denen ebenfalls der restringierte Code verwendet wird.

Kennzeichnend für die Sprache der Comics und der graphic novels ist die Verwendung der folgenden Elemente: Onomatopoetika, Inflektive, Mischformen aus Onomatopoetika, Inflektiven und Interjektionen. Sie alle erscheinen syntaktisch außerhalb des Satzzusammenhangs. Auch wenn diese nicht unbedingt zum aktiven Wortschatz der jungen Leser gehören, werden ihnen Bedeutungen zugemessen. Eine disambiguierende Hilfestellung bittet dabei der außensprachliche (grafische) Kontext. Es liegen Untersuchungen vor, aus denen hervorgeht, dass für die Sprache der Comics, bedingt durch das Medium, kürzere Sätze und Wörter prägend sind (SanchezStockhammer 2012). Dies liegt zum einen darin begründet, dass sich die Sprache an der Mündlichkeit orientiert, zum anderen aber erklärt sich die Reduktion aus den rein pragmatischen Überlegungen, denn längere Sätze und längere Wörter würden nicht mehr in die Sprechblasen passen und entweder die Textlastigkeit auf ein unästhetisches Maß erhöhen oder die Notwendigkeit schaffen, die Sätze auf mehrere Bilder aufzuteilen. Diese Besonderheit der Comicsprache und die Funktion des Sprachgebrauchs können zum gewinnbringend zum Gegenstand der unterrichtlichen Betrachtung gemacht werden. 


\subsection{Funktionspotenzial der Schrift und ihre Synästhetische Qualität}

Comics und graphic novels nützen beide Kommunikationskanäle, den graphischen und den schriftlichen: Schrift transportiert Sprache und somit Bedeutung, aber sie kann selbst ein grafisches Muster darstellen. Ferner kann sie als ein Teil der erzählten Wirklichkeit auftreten, also als ein diegetisches Element, beispielsweise in Form von Aufschriften auf Litfaßsäulen oder von den Figuren gelesenen Zeitungen. Es ist ein Spezifikum von graphic novels und Comics, dass der sprachliche und der grafische Aspekt der Sprache gleichwertig werden oder dass die grafische Seite sogar in den Vordergrund tritt (ein eindrucksvolles Bespiel sind die Arbeiten von Will Eisner). Das Visuelle vermittelt Sinneswahrnehmungen: unterschiedliche Lautstärken finden ihren Ausdruck in der Größe der Buchstaben. So stehen große Buchstaben für die erhöhte Lautstärke, während sehr kleine eine geringe - das Flüstern - darstellen. Den Lernenden dürfte diese Art der Darstellung aus Kommunikationskonventionen in sozialen Netzwerken bekannt sein. Die Tonhöhe lässt sich weniger eindeutig bestimmen, aber die einzelnen Variationen sind durchaus feststellbar, beispielsweise mittels wellenförmigen Buchstabenfolgen kann die sinkende Intonation abgebildet werden. Die verzerrten Lettern verhelfen zur Darstellung der Klangfarbe, beispielsweise der zitternden Stimme. Die endgültige Sinnzuschreibung und Bedeutungsgebung sind jedoch kontextbedingt. Auch der Fettdruck und Kursivierungen als suprasegmentale Elemente mit distinktiver Funktion können in dieser Funktion verwendet werden. Die Schriftgestaltung kann der Charakterisierung von situationsunabhängigen Sprechweisen von Figuren dienen, d.h. sie kann als ein Mittel zum Darstellen von individuellen oder regionalen Aussprachen verwendet werden (fakultative Varianten). Einen Gegenpol bilden die neutralen Darstellungen der typografischer Schrift. Aber auch dieser Neutralität kann eine Bedeutung zugewiesen werden - Korrektheit, Kühle, innere Ruhe oder Gleichgültigkeit in Abhängigkeit vom situativen Kontext. Es ist insofern Martin Schüwer zuzustimmen, dass die Schrift in einem Medium, in dem Sie grundsätzlich Gegenstand der Gestaltung ist, nie wirklich neutral sein kann (Schüwer 2012).

\subsection{Intermediale Vergleiche: Film}

Thematisiert man die Diversität der in Comics und graphic novels verwendeten Zeichen, so bietet es sich an, eine Parallele zum Medium Film $\mathrm{zu}$ ziehen. Anhand einzelner panels und gutters lässt sich der konnotative 
Mehrwert der Bildanteile (Koch 2009) eindrucksvoll demonstrieren. Anders als Filme ermöglichen die graphic novels jedoch eine verlagsamte Rezeption und eine verzögerte Wahrnehmung, die Aktivierung innerer Bilder unterstützt und dadurch zur Vorstellungs- und Verstehensleistung führt (Köppert 1999). Comics und graphic novels bringen Bilder zum Stillstand und erlauben dadurch das reflektierende Verweilen beim Einzelbild, die kontemplative Betrachtung einer Situation und Vor- und Rückgriffe zwischen den einzelnen panels und fördern ästhetische Erlebnisse. Aber es lassen sich ebenfalls viele Berührungspunkte zum Medium Film ausmachen. Hierzu zählen Elemente der Mikro- uns Makroanalyse - Analyse des Bildinhalts, der Einstellungsgrößen und der Bildgestaltung sowie Analyse narrativer Elemente, der Struktur, des Erzählers und der Zeitgestaltung.

\section{METHODISCHE VORGEHENSWEISE}

Die Thematisierung von graphic novels und Comics im Deutschunterricht lässt vielfältige methodische Zugriffe zu: analytische, handlungs- und produktionsorientierte und szenische Verfahren.

\subsection{Analytische Verfahren}

Neben der Analyse semiotischer Diversität bieten graphic novels zahlreiche authentische Anlässe zur Sprachreflexion einschließlich unterschiedlicher Sprachstile. Untersucht werden können Schauplätze und Orte, Farbgebung, Symbole, Verhältnis und Interdependenz von Bild- und Text, sowie die unterstützende Rolle der Visualisierungen im Verstehensvorgang. Ferner ist die Konkretisierung der Handlung und Figuren sowie Reflexion von thematischen und erzählstrukturellen Aspekten zu nennen. Die zeichnerische Darstellung erlaubt noch vor der unmittelbaren Lektüre Rückschlusse über die Charaktereigenschaften zu machen. Während und im Anschluss daran kann die Aufmerksamkeit sowohl auf die Selbstcharakterisierung als auch auf die Fremdcharakterisierung der Figuren gelenkt werden. Erfolgt die Auseinandersetzung mit den Protagonisten noch vor der Lektüre kann das antizipierende Lesen geübt werden. Die Schüler stellen Hypothesen über den Inhalt der graphic novel auf und spekulieren über die Handlung. Hier kann ein Anlass zur Förderung der Schreibkompetenz gesehen werden, indem die Lernenden eine Zusammenfassung für die mögliche Geschichte verfassen. 


\subsection{Handlungs- und produktionsorientierte Verfahren}

Im Rahmen des handlungs- und produktionsorientierten Unterrichts kann auf das gesamte verfügbare Repertoire von der Erstellung von Lesetagebüchern, über Art-Talk oder Plot/ Story-Rekonstruierungsaufgaben bis zur kollaborativen Erarbeitung in Kleingruppen zurückgegriffen werden. Bei den Plot-Rekonstruierungsaufgaben geht es um das Erfassen von Geschehens- und Handlungsverläufen. Hierfür können die einzelnen panels in die richtige Reihenfolge gebracht und/ oder die Sprechblasen ausgefüllt werden, oder aber die Schüler erhalten nur Bilder oder die Texte bzw. einen Text und erstellen dazu selbst eine visuelle Narration mit ihrem gesamten Inventar an Figuren, Schauplätzen, Dialogen und Interaktionen und berücksichtigen dabei die semiotische Verschiedenheit der einzelnen Zeichen. Bei der zuletzt beschriebenen Vorgehensweise müssen sie Entscheidungen über die sprachliche Gestaltung treffen und die längeren Sätze der textuellen Vorlage an die Spezifik des Mediums Comic anpassen.

Bei den rein produktiven Aufgaben absolvieren die Schüler mehrere Phasen von der Ideenfindung über Erstellung eines Exposés, bis zum Entwurf einer Dramaturgie. Sie wenden narratologische Kategorien an und wie bei der Produktion eines Films bedenken sie die Figuren und konkretisieren diese in Bezug auf das Äußere, Sprache, Gefühle und Gedanken sowie Handlungen. Einen weiteren Schwerpunkt bilden die Bestimmung einzelner Einstellungen und Perspektiven und schließlich der Entwurf eines Comics. Es sind Schauplätze und Orte zu bestimmen, sowie akustische Phänomene Verwendung von soundwords, die beim Rezipienten einen onomatopoetischen Eindruck evozieren, und Bewegung, die mittels der dafür vorgesehenen Indices und Symbole darzustellen ist. Es können Zusammenfassungen und Klappentexte als eine Art 'Vermarktungsstrategie' verfasst werden das wäre ein authentischer Anlass für die Förderung der Schreibkompetenz. Eingebunden werden kann das poetische, das klärende, das expressive und das kreative Schreiben (Abraham 2012).

Als ein weiteres Bespiel für die Ideenfindung kann die Arbeit mit den Zeitungen und mit den aktuellen Tagesereignissen sein oder aber auch Aufbereitung besonderer Begebenheiten oder autobiografischer Erlebnisse und Erfahrungen der Schüler. Es geht dabei nicht um die zeichnerische Versiertheit, wenngleich an dieser Stelle die Möglichkeit für den fächerverbindenden Unterricht im Fach Kunst gegeben ist. Einfache Formen der grafischen Darstellung, Hilfsmittel - beispielsweise einzelne vorgefertigte panels oder Anleitungen im Internet sind potenzielle Optionen. Entscheidend ist, dass diesen Vorgehensweisen die Erkenntnisarbeit vorangestellt wird, und die 
Schüler dadurch die Möglichkeit erhalten an der Comic-Kultur teilzuhaben und sich ihrer Konventionen und spezifischen Besonderheiten bewusst zu werden.

\subsection{Szenische Verfahren}

Szenische Verfahren erfreuen sich mittlerweile einer größeren Beliebtheit und haben ihren festen Platz in den Kerncurricula und somit im Deutschunterricht eingenommen. Auch bei der Arbeit mit graphic novels und Comics können diese zum Einsatz kommen.

\section{FAZIT}

Zusammenfassend lässt sich festhalten, dass graphic novels und Comics ein vielfältiges Potenzial für den Deutschunterricht haben. Sie eignen sich im Hinblick auf die Entwicklung und Förderung eines differenzierten Medienverständnisses aufgrund ihrer komplexen bildnerischen und sprachlich komprimierten strukturellen Beschaffenheit. Sie greifen Rezeptionsgewohnheiten Jugendlicher auf, da sie in ihrer Aspekthaftigkeit die Lebenswirklichkeit der Schüler wiederspiegeln und Ansätze der Integration von Medienrezeption, Mediennutzung und Medienreflexion bieten. Die Bereiche der Leseund der Schreibkompetenz, lexikalische und sprachreflexive sowie literarische Kompetenzen können erfolgreich gefördert werden. Bezieht man die ausländischen graphic novels und Comics mit ein, so ist auch das (inter)kulturelle Lernen möglich. Entscheidend ist, dass der Unterricht auf Erkenntnisarbeit und Erkenntnisgewinn ausgelegt ist, sei dies nun der Rezeptionsvorgang von Texten und Bilder an sich, die semiotische Diversität oder Funktionen der Schrift und ihre synästhetische Qualität oder die Konventionen und Spezifika der Comic-Kultur.

\section{LITERATURVERZEICHNIS}

Abraham, U. 2012. Filme im Deutschunterricht. Stuttgart: Klett Kallmeyer.

Dijk, T.A. van, Kintsch, W. 1983. Strategies of Discourse Comprehension. London: Academic Press Incorporated.

Eisner, W. 1998. Grafisches Erzählen. Graphic Story Telling. Wimmelbach: Comic Press Verlag.

Gadamer, H.-G. 1990. Hermeneutik: Wahrheit und Methode. Grundzüge einer philosophischen Hermeneutik. Gesammelte Werke. Band 1. Tübingen: Mohr Siebek. 
Gold, A. 2007. Lesen kann man lernen. Lesestrategien für das 5. und 6. Schuljahr. Göttingen: Vandenhoeck und Ruprecht.

Hallet, W. 2010. Viewing cultures: Kulturelles Sehen und Bildverstehen im Fremdsprachenunterricht. In Hecke, C., Suhrkamp, C. (Hrsg.). Bilder im Fremdsprachenunterricht. Neue Ansätze, Kompetenzen und Methoden. Tübingen: Narr, 26-54.

Hallet, W. 2012. Graphic Novels. Literarsches und multiliterales Lernen mit ComicRomanen. In: Der Fremdsprachlichen Unterricht Englisch, 117/ 46, 2-9.

Horus 2005. Schiller! Eine Comic-Novelle. Köln: Egmont.

Iser, W. 1975. Im Lichte der Kritik. In: Warning, R. (Hrsg.). Rezeptionsästhetik: Theorie und Praxis. München: Fink, 25-342.

Karg, I. Kuzminykh, K. 2014. Sprache und Literatur als Bildungskomponenten. Frankfurt a.M.: Peter Lang.

Kintsch, W. 1998. Comprehension. A Paradigm for Cognition. Cambridge: University Press.

Koch, P. Oesterreicher W., 1985. Sprache der Nähe - Sprache der Distanz. Mündlichkeit und Schriftlichkeit im Spannungsfeld von Sprachtheorie und Sprachgeschichte. In: Romantisches Jahrbuch, 36, 15-43.

Koch, S. 2009. Literatur - Film - Unterricht: Bewertungsgrundlagen und didaktisches Potenzial der Literaturverfilmung für den Deutschunterricht am Beispiel „Eyes Wide Shut”. Würzburg: Königshausen \& Neumann.

Köppert, C. 1999. Innere Bilder zu laufenden Bildern. In: Praxis Deutsch, 154, 53-59.

Kreitz, I. 2009. Pünktchen und Anton. Hamburg: Dressler.

Kreitz, I. 2012. Emil und die Detektive. Hamburg: Dressler.

McCloud, S. 2001. Comics richtig lesen. Hamburg: Carsen.

Monaco, J. 2000. Kunst, Technik, Sprache, Geschichte und Theorie des Films und der Medien. Mit einer Einführung in Multimedia. Reinbek bei Hamburg: Rowohlt.

Monnin, K. 2009. Teaching Graphic Novels. Gainesville: Maupin House Publishing.

Pierce, C.S. 1931-1958. Collected Papers 1-8. Cambridge: Haravrd University Press.

Sanchez-Stockhammer, C. 2012. Comicsprache - leichte Sprache? In: Pietrini, D. (Hrsg.) Die Sprache(n) der Comics. Heidelberg: Martin Meidenbauer, 55-75.

Schüwer, M. 2008. Wie Comics erzählen. Grundriss einer intermedialen Erzähltheorie der graphischen Literatur. Tier: WVT.

Schüwer, M. 2012. Laute malen: Zum Status der Schrift in Comics. In: Pietrini, D. (Hrsg.). Die Sprache(n) der Comics. Heidelberg: Martin Meidenbauer, 15-33.

Welke, M. 1972. Die Sprache der Comics. Frankfurt a.M.: dipa. 